\begin{tabular}{|l|l|l|l|l}
\hline Revue suisse Zool. & Tome 90 & Fasc. 4 & p. 899-905 & Genève, décembre 1983
\end{tabular}

\title{
Le genre Plusiocampa dans les grottes sardes (Diploures Campodéidés)
}

par

B. CONDÉ *

Avec 4 figures

ABSTRACT

The genus Plusiocampa in Sardinian caves (Diplura Campodeidae). - Description of Plusiocampa socia n. sp., from the cave "Cane Gortoe" in Sardinia, the second species of the genus known from the island. It is compared with more or less related species from Majorca, Italy and southern France. A key for eight species or subspecies in included.

Les premiers représentants sardes du genre Plusiocampa s.str., ont été découverts dans la grotte de San Giovanni, près de Domusnovas, dans le sud-ouest de l'île, par le très regretté Marchese Saverio Patrizi Montuoro, en août 1952. Ils étaient abondants sur les bancs d'argile humide dans la portion sud de la galerie secondaire. Les 31 spécimens (11 mâles, 11 femelles et 9 immatures) qui me furent communiqués furent rapportés, en 1953, à l'espèce continentale Flusiocampa provincialis Condé, 1949, en suggérant qu'ils pourraient appartenir en fait à une sous-espèce distincte. De nouvelles observations m'ont conduit à leur attribuer, en 1981, un rang spécifique: Plusiocampa sardiniana.

Une seconde espèce de Plusiocampa s.str. fut récoltée, également par S. Patrizi, dans la grotte Cane Gortoe, à Siniscola, sur la côte orientale, en juin 1955. Une visite de cette cavité, le 6 octobre 1955, en compagnie de H. Henrot et S. Patrizi, ne permit pas de retrouver cette intéressante espèce. L'unique spécimen connu fut cité sous Plusiocampa socia n. sp. (CONDÉ 1956: 199), en mentionnant seulement ses affinités avec $P$. fagei Cdé, de Majorque et $P$. romana Cdé, de la péninsule italienne. $P$. socia doit donc être considéré jusqu'à ce jour comme un nomen nudum.

* Université de Nancy I, Zoologie approfondie, 34, rue Sainte-Catherine, F-54000 Nancy, France. 
Nous nous proposons de donner ici une description aussi complète que possible de l'holotype de $\boldsymbol{P}$. socia et de situer cette espèce par rapport à ses voisines insulaires ou continentales à l'aide d'une clé récapitulant les principaux caractères utilisés.

La mise au propre des illustrations a été exécutée par $\mathbf{M}^{\mathrm{me}} \mathrm{C}$. Langton.

\section{Plusiocampa (s. str.) socia n. sp.}

\section{(Plusiocampa socia Condé, 1956, nomen nudum)}

MatéRIel. - Prov. Sassari. Grotta di Cane Gortoe à Siniscola, dans le village de ce nom, s'ouvrant sur une propriété enclose, rivière souterraine peu profonde, mais très longue; sur l'argile, à $200 \mathrm{~m}$ environ de l'entrée, S. Patrizi, 14-VI-55: 1 ㅇ jeune, longue de $5,5 \mathrm{~mm}$, sans les cerques qui manquent (holotype). Montée sur 2 lames dans le médium II de Marc André. Muséum de Genève, Département des Arthropodes et Insectes II.

LONGUEUR. - 5,5 mm, sans les cerques qui manquent.
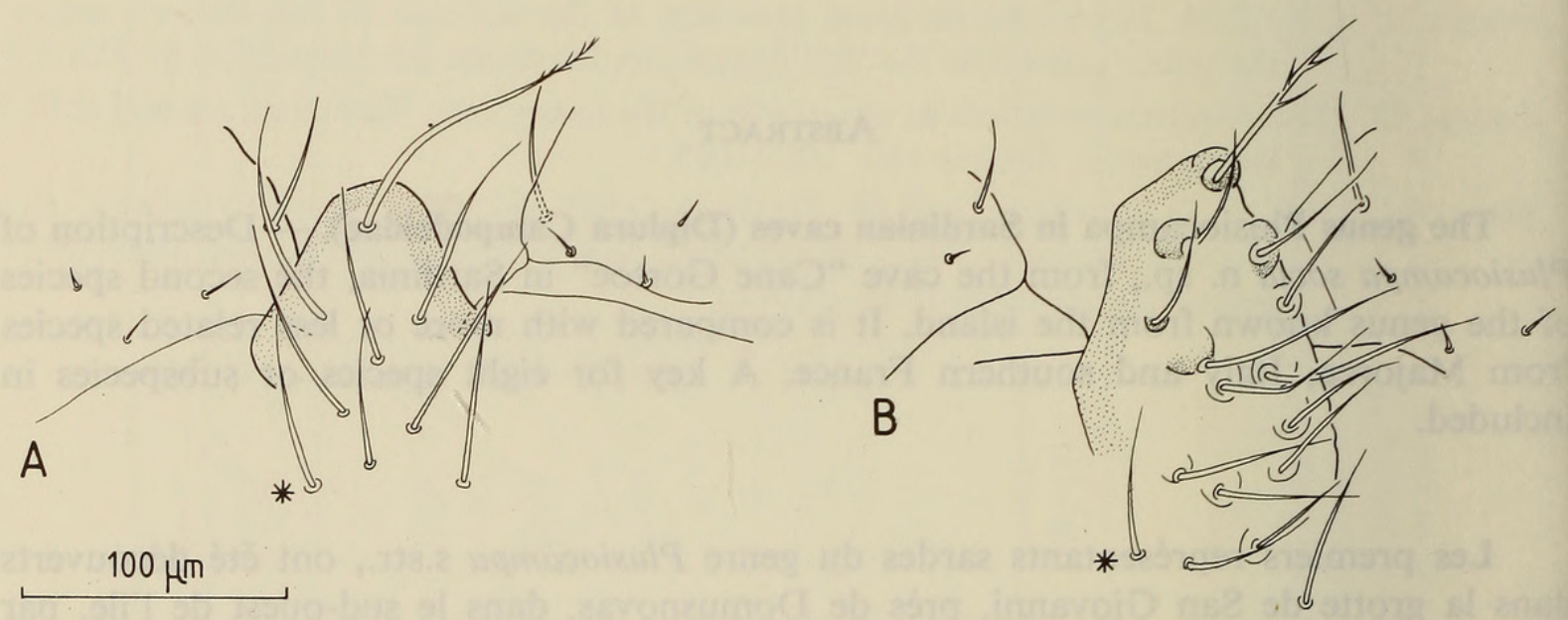

FIG. 1.

Plusiocampa socia n. sp.. Femelle holotype. A. Processus frontal; Plusiocampa sardiniana Condé. Femelle paratype. $B$. Processus frontal. L'astérisque indique l'homologie probable des phanères.

TÉGUMENTS. - Epicuticule dépourvue d'ornementation. Soies de revêtement glabres, quelques unes avec des barbules minuscules et peu nombreuses sur la région terminale; celles des segments IX et $\mathrm{X}$ semblables à celles des segments précédents.

TÊTE. - Antenne gauche, seule complète, de 36 articles, l'apical étant très allongé (environ 110/27), ce qui porte à croire qu'il s'agit d'un régénérat. Article III avec un sensille bacilliforme postéro-sternal, court et grêle, entre $d$ et $f$ (tout près de $d$ ), ses longs macrochètes barbelés sur leur portion distale. Organe cupuliforme de l'article apical renfermant 9 sensilles ( 7 périphériques et 2 centraux) de forme complexe.

Processus frontal avec un seul macrochète apical long (62), mais très peu barbelé, et 5 soies proximales grêles, aucune embase n'étant tuberculée. Macrochètes bordant 
la ligne d'insertion des antennes de longueurs un peu inégales ( $a=42,5, i=46,5$, $p=39$ ) et faiblement barbelés à l'apex; macrochètes $(x)$, insérés un peu en avant des branches latérales de la suture en $Y$, les plus différenciés (69); macrochètes occipitaux médiaux moyens (48) et latéraux postérieurs plus développés (56-65).

THORAX. - Répartition des macrochètes tergaux:

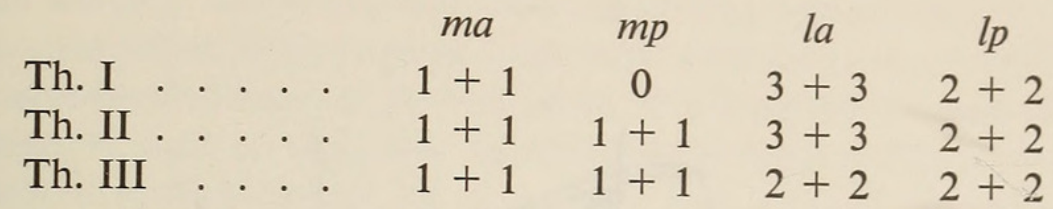

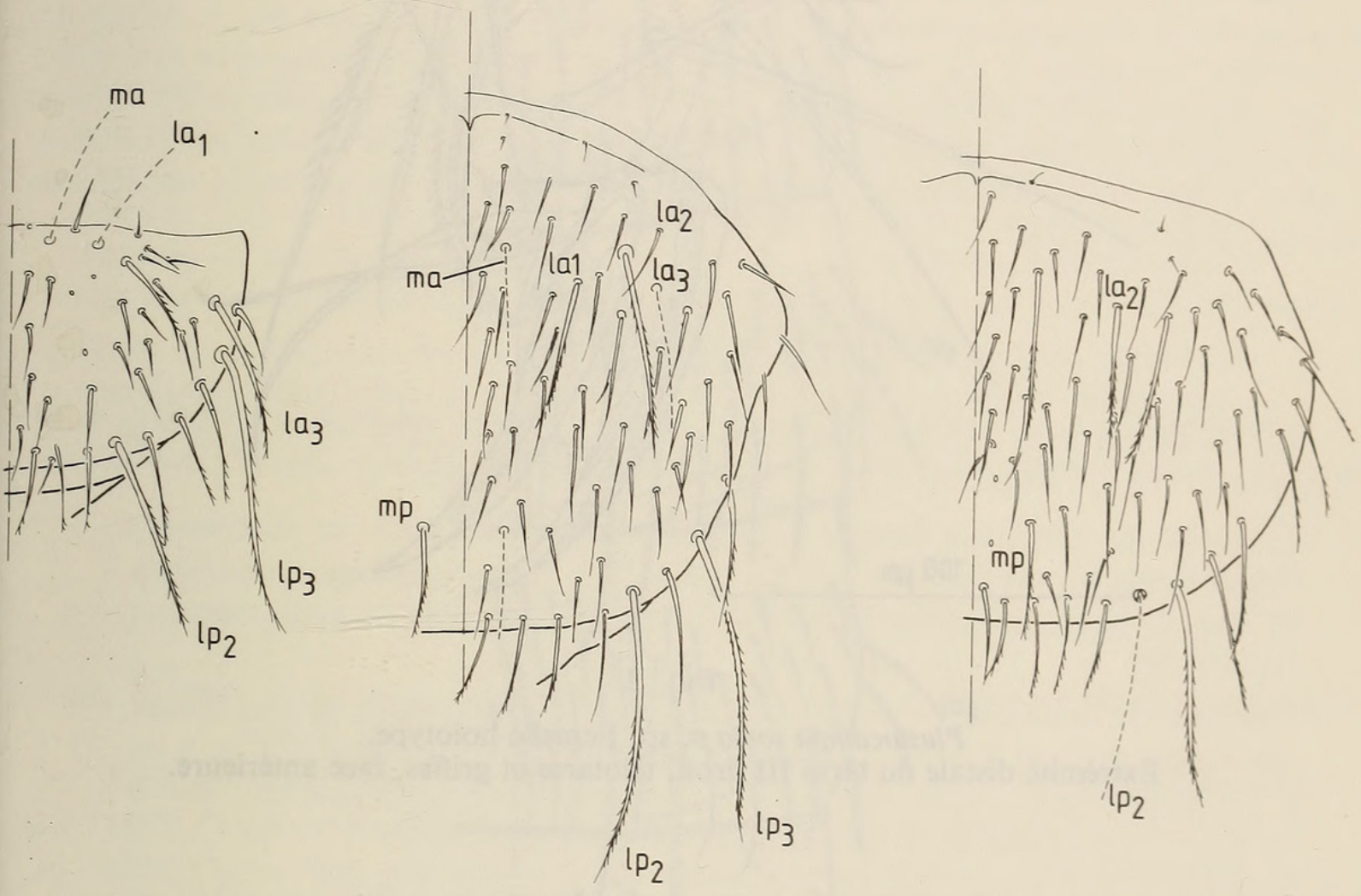

FIG. 2.

Plusiocampa socia n. sp. Femelle holotype. Pro-, méso- et métanotum.

Ces phanères dont plusieurs manquent bilatéralement ( $m a$ et $l a_{3}$ au mésonotum, $l p_{2}$ au métanotum) sont longs et grêles, barbelés sur leurs $2 / 3$ à $3 / 4$ distaux, 3 à 5 soies marginales postérieures par demi-tergite séparent les $l p_{2}$. Les macrochètes médiaux postérieurs $(\mathrm{mp})$ méso- et métanotaux sont semblables entre eux, les métanotaux étant 1 fois $1 / 2$ plus courts que les $m a$ correspondants (70/103); ce sont les plus courts et les plus grêles des macrochètes.

Pattes allongées et grêles, les métathoraciques atteignant presque le bord postérieur du VIII e segment. Le macrochète tergal du fémur III est inséré un peu au-delà du milieu du bord tergal de l'article (63/104), sa longueur étant égale au $1 / 3$ de ce bord (35/104). Tibia avec 2 macrochètes sternaux. Phanères du tarse barbelés, au moins sur leur portion 
proximale, à l'exception des sensilles sétiformes, de rares soies de revêtement et des 3 grands phanères subapicaux tergaux qui sont entièrement glabres. Soie subapicale antérieure et soies subapicales sternales barbelées sur toute leur longueur. Griffes très légèrement inégales (105/109) à crêtes latérales ornementées, quoique de dimensions modestes; processus télotarsaux sétiformes et glabres.

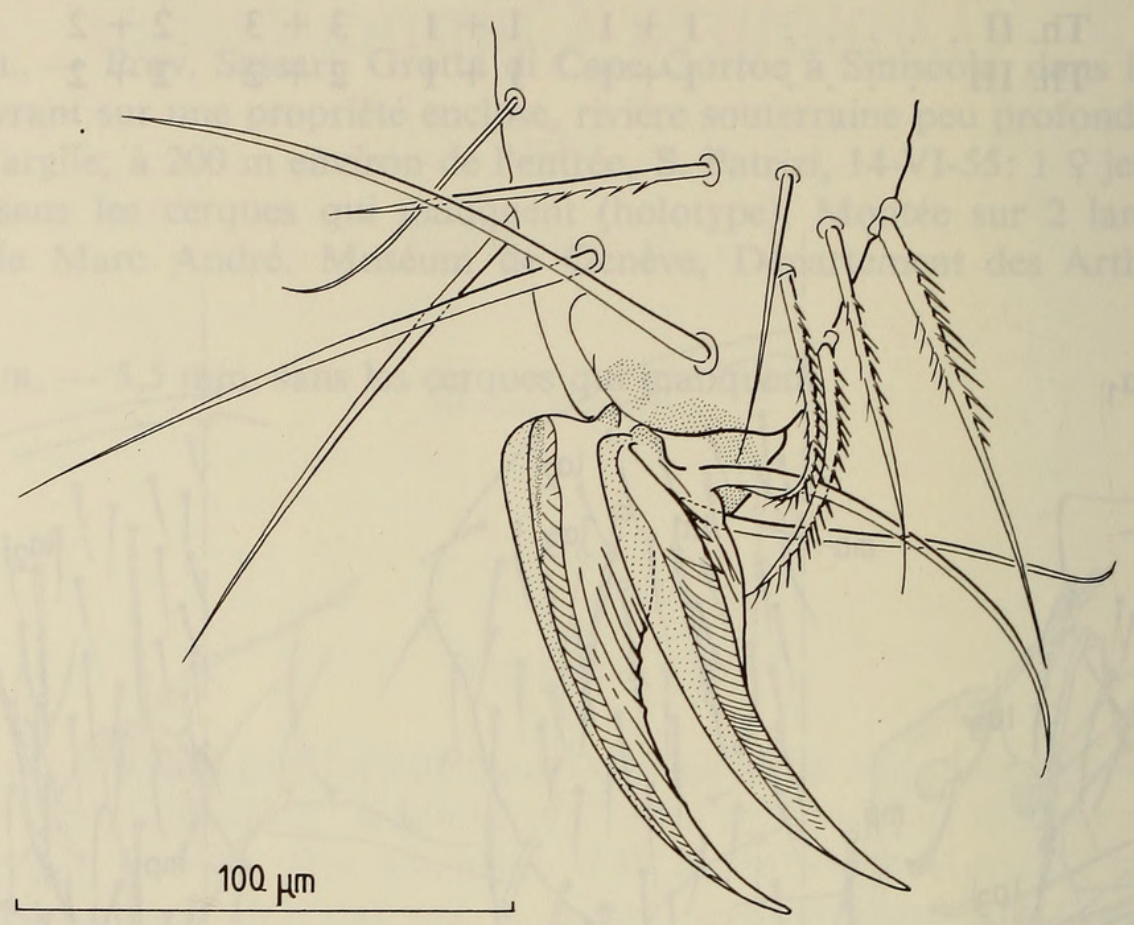

FIG. 3.

Plusiocampa socia $\mathrm{n} . \mathrm{sp}$. Femelle holotype.

Extrémité distale du tarse III droit, télotarse et griffes, face antérieure.

AвDOMEN. - Répartition des macrochètes tergaux:

\section{la}

Ab. I

Ab. II

Ab. III

Ab. IV

Ab. V

Ab. VI-VII . . . . . . . . .

Ab. VIII .

$$
\begin{gathered}
0 \\
0 \\
0+1\left(l a_{3}\right) \\
1+1 \\
1+1
\end{gathered}
$$$$
2-3+2-3\left(l a_{2,3}-l a_{1,2,3}\right)
$$

0 post

$2+1\left(\right.$ post $\left._{1,3}\right)$

$2+2$

$2+2$

$2+2$

$4+4\left(\right.$ post $\left._{1,3,4,5}\right)$

$4+4$

$6+6$

Les macrochètes de la paire la plus voisine du plan sagittal ( post $_{1}$ ) sont séparés l'un de l'autre par 5 ou 6 soies marginales aux tergites I-II et 6 à 8 aux tergites III-VIII.

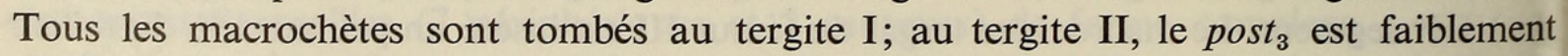
différencié à droite, 2 fois plus court que le post $t_{1}(65 / 131)$, et tombé à gauche; au tergite III, ils sont encore un peu plus courts que les post p $_{1}(112 / 136)$; à partir du tergite IV, 
les post $_{3}$ sont au contraire un peu plus longs que les post 1 (172/166). Valvule supraanale avec une seule soie subapicale.

Plaque sternale I avec $8+7$ macrochètes bien différenciés; le macrochète surnuméraire asymétrique latéral antérieur est régulièrement présent chez $P$. fagei; ses appendices sont subcylindriques, avec 9 poils glandulaires apicaux $\left(a_{1}\right)$. Sternites

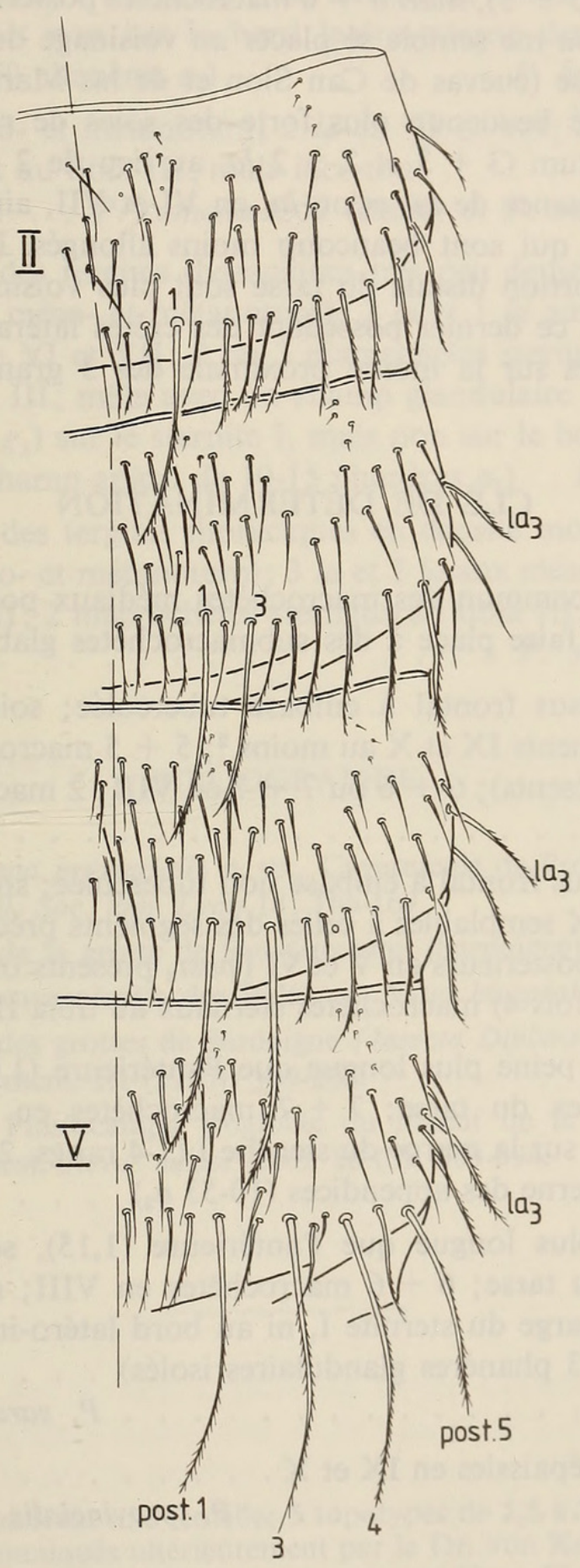

FIG. 4.

Plusiocampa socia n. sp. Femelle holotype. Tergites abdominaux II à V. 
II-VII avec $5+5$ macrochètes, VIII avec $2+2$. Volets génitaux avec chacun une rangée submarginale de 4 soies; tubercule impair avec $6+6$ soies.

Styles comme chez $P$. fagei Condé.

Affinités. Plusiocampa socia est facile à distinguer de $P$. sardiniana Cdé qui est le seul autre représentant du genre connu de l'île et appartient au complexe de $P$. provincialis Cdé. A ce titre, sardiniana possède des soies de revêtement épaisses et fusiformes sur les segments IX et $\mathrm{X}$, des phanères frontaux à embase tuberculée, des post $_{2}$ aux tergites IV à VII au moins $(5+5)$, mais $6+6$ macrochètes postérieurs seulement en VIII ${ }^{1}$.

En revanche, $P$. socia me semble se placer au voisinage de mon $P$. fagei, décrit de deux grottes de Majorque (cuevas de Can Sion et de las Maravillas). Il s'en distingue cependant par la densité beaucoup plus forte des soies de revêtement, la chétotaxie du méso- et du métanotum $(3+3$ et $2+2$ la, au lieu de $2+2$ et $1+1)$, l'absence de post $_{2}$ en VII et la présence de $l a_{1}$ et/ou $l a_{2}$ en VI et VII, ainsi que par la forme des appendices du sternite I qui sont beaucoup moins allongés. Les caractères des griffes et des phanères de la portion distale du tarse sont plus voisins de ceux de $P$. romana que de ceux de $P$. fagei, ce dernier possédant des crêtes latérales beaucoup plus développées et des barbelures sur la moitié proximale des 3 grands phanères subapicaux tergaux (1955, fig. 3D).

\section{CLÉ DE DÉTERMINATION}

Les espèces ont en commun des macrochètes médiaux postérieurs au méso- et au métanotum qui peuvent faire place à des submacrochètes glabres chez $P$. magdalenae.

1. Phanères du processus frontal à embase tuberculée; soies de revêtement épaissies sur les segments IX et $\mathrm{X}$ au moins ${ }^{2} ; 5+5$ macrochètes postérieurs en V et VI ( post $_{2}$ présents); $6+6$ ou $7+7$ en VIII; 2 macrochètes sternaux au tibia III

- Phanères du processus frontal à embase non tuberculée; soies de revêtement des segments IX et $\mathrm{X}$ semblables à celles des segments précédents; $5+5$ ou $4+4$ macrochètes postérieurs en V et VI ( post $_{2}$ présents ou absents); $6+6$ en VIII; 2 ou 3 (parfois 4) macrochètes sternaux au tibia III . . . . . . .

2. Griffe postérieure à peine plus longue que l'antérieure $(1,06)$, son talon ne recouvrant pas l'apex du tarse; $7+7$ macrochètes en VIII; mâle avec champs glandulaires sur la marge du sternite I (1-4 rangs, 23 à plus de $\left.60 g_{1}\right)$ et au bord latéro-interne des appendices $\left(10-53 a_{2}\right)$. . . . . . . . . . .

- Griffe postérieure plus longue que l'antérieure $(1,15)$, son talon saillant recouvrant l'apex du tarse; $6+6$ macrochètes en VIII; mâle sans champ glandulaire sur la marge du sternite I, ni au bord latéro-interne des appendices (au plus 2 ou 3 phanères glandulaires isolés) .

P. sardiniana Cdé (Sardaigne)

3. Soies de revêtement épaissies en IX et X . . . . . . . . . . . . . . . .

$P$. provincialis Cdé, f. typ. (France)

${ }^{1}$ Il y en a $7+7$ et non $6+6$, comme il est indiqué par erreur dans la description originale, chez $P$. provincialis (1949: 167).

${ }^{2}$ L'appréciation de ce caractère est particulièrement facile en examinant la face sternale. 
- Soies de revêtement épaissies en VIII, IX et X

$P$. provincialis praedita Cdé. (France)

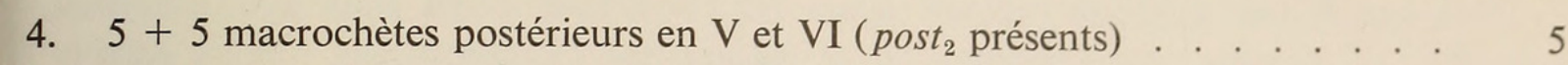

- $4+4$ macrochètes postérieurs en V et VI (post $t_{2}$ absents) . . . . . . . . 6

5. 2 la (une fois $3+2$ ) et 1 la aux méso- et métanotum; 2 la en VI et VII; 3 (excep. 4) macrochètes sternaux (dans la proportion 6/2) au tibia III; mâle avec un champ glandulaire très large (une dizaine de rangs, 315 phanères $g_{1}$ ) sur le sternite I, mais non sur le bord latéro-interne des appendices (un champ apical de 40-50 phanères $a_{1}$ ) . . . . . . . . P. breuili Cdé ${ }^{3}$ (Majorque)

- 3 la et 2 la aux méso- et métanotum; 3 la en VI et VII; 2 ou 3 (excep. 4) macrochètes sternaux au tibia III; mâle inconnu.

$P$. romana Cdé (Italie) et $P$. magdalenae Cdé (France)

6. Soies de revêtement des tergites thoraciques très peu denses ( 6 et 3 au plus par demi-tergite aux méso- et métanotum); 2 la et 1 la aux méso- et métanotum; un seul la en VI et VII; 2 ou 3 macrochètes sternaux (dans la proportion 5/3) au tibia III; mâle avec un champ glandulaire étroit (2-3 rangs, environ 68 phanères $g_{1}$ ) sur le sternite $\mathrm{I}$, mais non sur le bord latéro-interne des appendices (un champ apical de 10-15 phanères $a_{1}$ ) . P. fagei Cdé (Majorque)

- Soies de revêtement des tergites thoraciques en densité moyenne (40-50 par demi-tergite aux méso- et métanotum); 3 la et 2 la aux méso- et métanotum; 2 ou 3 la en VI et VII; 2 macrochètes sternaux au tibia III; mâle inconnu

$P$. socia n. sp. (Sardaigne)

\section{BIBLIOGRAPHIE}

CondÉ, B. 1949. Plusiocampa provincialis n. sp., Cavernicole de Provence (Diploures Campodéidés). Bull. Soc. linn. Lyon 18: 166-169.

- 1953. Campodéidés de la grotte de San Giovanni (Sardaigne). Notes biospéol. 8: 33-37.

- 1955. Campodéidés cavernicoles des Baléares. Notes biospéol. 9: 121-132.

- 1956. Campodéidés des grottes de Sardaigne (Insecta Diplura). Ras. speleol. ital. e Soc. speol. ital., mem. III (1955): 199-202.

- 1981. Un nouveau Plusiocampa trogiobie du massif de la Sainte-Baume (Diploures Campodéidés). Revue suisse Zool. 88 (3): 589-594.

${ }^{3}$ L'holotype de $P$. breuili est une femelle; 5 topotypes de 5,5 à $8 \mathrm{~mm}$, parmi lesquels un $\hat{o}^{\top}$ de 5,5 mm, m'ont été communiqués ultérieurement par le Dr. von Keler (Humboldt-Universität zu Berlin), récoltés par Bischoff (IX-1958). Les antennes ont 42 ou 43 articles (holotype: 30 et 32) et au moins 22 sensilles dans l'organe cupuliforme; les griffes et les processus télotarsaux ne sont pas émoussés et raccourcis comme chez le type, ce qui était peut-être la conséquence d'une usure anormale; 5 à 10 barbules sternales sur la moitié proximale des processus télotarsaux; le tibia III droit du $\sigma^{\hat{t}}$ et d'une $q$ porte 4 macrochètes sternaux. 


\section{$2 \mathrm{BHL}$ Biodiversity Heritage Library}

1983. "Le genre Plusiocampa dans les grottes sardes (diploures campodeides)." Revue suisse de zoologie 90, 899-905. https://doi.org/10.5962/bhl.part.82014.

View This Item Online: https://www.biodiversitylibrary.org/item/128827

DOI: https://doi.org/10.5962/bhl.part.82014

Permalink: https://www.biodiversitylibrary.org/partpdf/82014

\section{Holding Institution}

Smithsonian Libraries

\section{Sponsored by}

Biodiversity Heritage Library

\section{Copyright \& Reuse}

Copyright Status: In Copyright. Digitized with the permission of the rights holder.

Rights Holder: Muséum d'histoire naturelle - Ville de Genève License: http://creativecommons.org/licenses/by-nc-sa/3.0/ Rights: https://www.biodiversitylibrary.org/permissions/

This document was created from content at the Biodiversity Heritage Library, the world's largest open access digital library for biodiversity literature and archives. Visit BHL at https://www.biodiversitylibrary.org. 